EDUCATIONAL

RESOURCE

Volume 10 Issue 22018

DOI: 10.21315/eimj2018.10.2.7

ARTICLE INFO

Submitted: $27-03-2018$

Accepted: 01-05-2018

Online: 29-06-2018

\title{
Palliative Care Approach for Preemie - An Ethical Case Learning
}

\author{
Fahisham Taib', Lee Chee Chan² \\ ${ }^{1}$ Department of Paediatrics, School of Medical Sciences, Universiti \\ Sains Malaysia, Kelantan, MALAYSIA \\ ${ }^{2}$ Department of Paediatrics, Hospital Kuala Lumpur, Kuala Lumpur, \\ MALAYSIA
}

To cite this article: Taib F, Lee CC. Palliative care approach for preemie - an ethical case learning. Education in Medicine Journal. 2018;10(2):55-59. https://doi.org/10.21315/eimj2018.10.2.7

To link to this article: https://doi.org/10.21315/eimj2018.10.2.7

\begin{abstract}
Palliative care for life limiting conditions usually starts at birth. In neonatal period, planning, discussion and goal of care should focus towards improving the baby quality of life. It does not mean palliative care in this age group mainly as end of life care. We illustrate a complex 30 weeks baby who was born with genetic abnormality complicated with intracranial bleeding and acute myeloid leukaemia. There were various ethical issues related to the approach of death and dying infant and psychosocial challenges surrounding the case.
\end{abstract}

Keywords: Prematurity, End of life, Ethics, Paediatrics, Life limiting

Fahisham Taib, Department of Paediatrics, School of Medical Sciences, Universiti Sains Malaysia, 16010 Kubang Kerian, Kelantan, Malaysia | Email: fahisham@gmail.com

\section{INTRODUCTION}

Palliative care in antenatal and neonatal period is a new area of interest in paediatric specialty. It covers the entire milieu of care to prevent and relieve infant suffering and improve the conditions of the infant's living and dying (1). This includes team approach towards common goal of relieving physical symptoms of the infants and existential suffering of the family. Premature babies are new-borns from the very early gestational periods of 22 to 24 weeks until less than 37 week, and may require neonatal intensive care admission. Aspect of palliative care includes appropriate intervention of physical, psychosocial, emotional and spiritual values and supporting family experience with empathy and sensitivity towards their needs. Supporting the family and siblings is an essential intervention as they go through life changing crisis and stressful period. Clinical areas deemed important are principle of palliative care, information of available services and support, crisis intervention and strategy to cope with stress, conflict mediation and cultural expectation.

\section{CASE SUMMARY}

$\mathrm{AF}$ is a 30 -weeker baby who was born by spontaneous vaginal delivery. $\mathrm{He}$ was quickly intubated for mild respiratory distress syndrome. The neonatal team noticed that AF has subtle dysmorphic features with genital anomaly. There was also marked bruises over the trunk and limbs following the delivery. Upon admission to neonatal intensive care (NICU), AF started to have seizure 
activities needing two antiepileptic drugs. Magnetic resonance imaging (MRI) head revealed extensive intracranial haemorrhage with bloods showing features of anaemia, thrombocytopaenia, hyperleukocytosis and coagulation abnormality. He had had bone marrow aspiration and was confirmed to have Acute Myeloid Leukaemia (AML). The haematologist suspected that AF may have an underlying genetic disorder such as Noonan syndrome. The mother was known to have depression in the past and unable to cope with the news. She stated that this was a precious pregnancy as she enters her 40th birthday recently. AF's father accepted the news and willing to do the best for his only child. Sadly, AF passed away following conservative medical management on neonatal intensive care unit.

\section{ISSUES}

The are several factors pointing towards difficult decision making in this scenario - early intracranial bleeding, hyperleukocytosis secondary to AML, underlying genetic abnormality with dysmorphism and prematurity. The baby was at risk of possible tumour lysis syndrome, with anticipated hyperviscosity leading to vaso-occlusive crisis and fragile neonatal organs. In the early stage, curative intent is necessary for a 30-weeker baby due to higher chance of survival. Evolving understanding with available information on the complexity of the case has somehow helped prognosticating the case. The initial impression of possible infantile leukaemia was either juvenile monocytic myeloid leukaemia (JMML), which carried better treatment outcome, or AML which has a guarded outcome. Chemotherapy in this case would be tricky due to little experience in administering chemotherapy to premature infant and risking subsequent damage to organs' function in a growing premature baby. This baby is also at risk to get infection due to weak immune system. In contrast, prognostication for children with nononcology life limiting conditions can be challenging due to uncertainties of the disease trajectory. Many of these children require active palliative care when needed, and the requirement fluctuate over time depending on the crisis points or child's needs.

Application of ethical principles such as beneficence and non-maleficence is important to avoid "heroic" attempt on any life limiting condition.

Careful consideration when relaying sensitive information to all family members is vital. Initiation of the discussion should be handled by leading team, which was the neonatal team. Paediatric palliative care and oncology would be the secondary care team members which manage the patient in parallel to the main team. Communication with the family members need to be opened so that they would be able to digest the information appropriately. The conversation with mother was deferred. This is primarily because mum felt that she was not coping with the news. Any decision made by mum was respected considering the knowledge on her fragile mental state and health. Capacity to understand the condition, the process of decision making, and intervention are a necessary component to ensure the family is not colluding information due to mother's mental state.

\section{Decision to Resuscitation}

Difficult conversation on resuscitation should be initiated. Our society lives in a notion to resuscitate at all cost. Paediatric Advance Care (PAC) plan is not the same as "do not resuscitation" order because the document is not a legal binding order. Parents still have time to reflect and change decision based on their wishes, understanding and preferences. Parents act as "proxy" decision maker, obviously their emotion and best interest choice should be respected. Parents are those who loved their children dearly, knowing their needs and wanting the best for their children. In the event where different intractable and 
irreconcilable difference between parents and physician occur, court ruling would be the final option to decide on child best interest after the physicians exhaust all avenues of negotiation (2). Negotiation is important to ensure good communication and relationship between doctor and parents. If this is not possible, ethical decision could go through other committee such as hospital ethics, legal team or private negotiators. The concept of "allowing natural death" may be suitable in the event where hope is loss, but this need to be cautiously and sensitively used during the time of discussion.

\section{Psychosocial Dilemma and Wishes}

The palliative goals for this scenario should be based on patient's quality of life and relieving suffering (3). The challenge when assessing neonates is the ability to assess them objectively on symptoms that cause suffering and distress. As we are aware, baby $\mathrm{AF}$ was ventilated and requiring supportive medical treatment for complication of the disease. Using strong chemotherapy may be contraindicated as we do not have tangible evidence to state that chemotherapy is beneficial and safely used in premature infants. Aside from that parallel planning regarding management for seizure, bleeding and issues related to parent's health should be discussed. Parents would require psychosocial support as well as psychological intervention in a difficult scenario such as baby AF. Time is needed for the parents to digest and absorb all the information about their children.

\section{Identifying Death and Dying Child}

Difficult conversation may revolve around the process of death and dying. It is often trying to reassure parents about the normality of the process so that they won't be surprise with the symptoms. According to Royal College of Paediatrics and Child Heath (RCPCH) document in 1997, regarding withholding and withdrawing treatment, five scenarios exist before decision can be made (4). Decision making would be based on the scenario whether it is - unbearable, no chance, no purpose, brain dead or permanent vegetative state. Although some of these conditions may be speculative, futility of medical care should trigger discussion to take place. The new $\mathrm{RCPCH}$ document sets the discussion on circumstances under which withholding or withdrawing life sustaining treatment might be ethically permissible to avoid confusion and misunderstanding (2).

\section{Forecasting Future - Supportive Care}

Due to mother's mental health issue, it is important to ensure available support to mum from the community team. If the hypothetically this child has a genetic condition and currently in a poor state, the trigger or crisis point should tilt the family to seek for psychological or spiritual help. Even if the child survives, there are other issues in relation to gender identity, disability, mental health and many other emotional related issues. We acknowledge that the child is a precious one as mum has nearly reached her sterility. Family dynamic would be important to ensure mum is kept on supported. But this interaction should be sensitive by giving space and time for parents to rethink and evaluate their decision and actions.

\section{ETHICAL JUSTIFICATION}

Most of the decisions in palliative care require careful consideration of patient's or parent's preferences and values, should they have capacity to understand the treatment and management of the related medical treatment. Unilateral decision making by the physician on the parent who have capacity to make consent can be dangerous and controversial. Medical futility is a common justification but the terms have different meaning such as infective treatment, in appropriate intervention which may lead for unfavourable benefit-harm ratio (5). Decision in this scenario would require 
medical expertise and value judgement due to incompetent minor with proxy decision making. The first part is to determine the chance of successful treatment in this condition. The goal of treatment should focus on curative intent and predicting the outcome of uncertain treatment would be difficult. Even if the curative intent is less than $1 \%$, the nature of information relayed to parents may influence the outcome of the management. One may have to differentiate between, if treatment given, the chance of cure is impossible versus the treatment is at risk of harm due to certainty of disease trajectory and progression.

Physician may assess difficult decision from the concept of benefit-harm ratio and best interest of the patient. This entails the principle of beneficence and non-maleficence. If harm likely to outweigh benefit, it is essential approach to intervention should be related to the reality of the condition. With factual events, often physician should be able to indicate trajectory of the illness accordingly. Certainly, communication is important in relaying prognostication, estimation of survival or palliative care goals. The consequences of inappropriate discussion and clinical decision may lead to collapsing trust between doctor-parent relationships. The other important aspect is the involvement of financial coverage in rare disease like. It is important not to underestimate the hospital potential restriction in distributing resources because at time, financial consideration may be crucial factor to consider. That does not mean, to state that, all palliative care patients are less important and hence receive less sophisticated approach to care. In controversial juncture of decision making, clinicians should allow information and discussion openly to achieve a common goal of treatment.

Clinicians owe moral responsibility to their patients based on best interest by respecting and preserving life, and respecting patients' decision without manipulation, coercion or deceiving the patients (2). Parents have ethical and legal authority to make the informed decision based on the best interest of the child. Parents' special relationship and their position in the society make them a vital role in decision maker, partnering with the clinicians. Right of the child, according to United Nation Convention on the Right of a Child (UNCRC), would include acting by the best interest as primary consideration, receiving high standard of care and respecting the child's view (6). $\mathrm{RCPCH}$ document discussed the potential situations where withholding or withdrawing treatments are possible. This has to be looked upon the quality of life rather than quantity due to potential differences in views on what constitute quality of life and the values it contains (2). It is important to acknowledge the importance of parents upon their child's life and their view of the quality for that child. The context where the discussion may go involves the burdens of the child's illness, the treatments that the child is already receiving and lack of ability to derive benefit.

Neonatal palliative care has been offered to babies with complex and life threatening medical conditions with uncertain prognosis, and its goal is to improve the quality of life. Most of the approaches circulate on end of life rather than life prolonging measures. This does not address the need of psychosocial intervention. Unfortunately, term such as palliative care is associated with giving up or losing hope (3). There is other aspect of assessment which relates to family spiritual assessment to derive family's hopes and dreams. This should be done in the context of parallel planning among the multidisciplinary team involvement. For example, in this scenario neonatal team would manage medical issue of the child, oncology to manage various difficult management haemato-oncology sequalae and palliative care for ongoing discussion on difficult decision. Ongoing care to support during the crisis episodes together with bereavement period can be a valuable approach and should be based on individualised family's plan. 
Mental health could be a challenging factor when breaking bad news. Support from the family is important to allow natural grieving process. The team is quite aware the potential consequences when hearing this news. Depression and stress would predispose mother to be able to make thoughtful clinical judgement. Capacity of making decision in adult should be based on understanding the whole medical process either withholding or withdrawing treatment. Concern on maternal health is highlighted, as well as potential effect of the condition to her child. It is important to remind the family on the direction of care which focusing on improving quality of life, spending precious moment together and aiming for realistic goals before the death of the loved one.

\section{CONCLUSION}

In neonate, approach to palliative care would follow similar principle. Family, sibling, bereavement care should be an ongoing approach once agreeable steps has been taken. Clarity of information with multidisciplinary approach would be a way forward in managing preemie with devastating and life limiting medical conditions.

\section{REFERENCES}

1. Catlin A, Carter B. Creation of a neonatal end-of-life palliative care protocol. Journal of Perinatology. 2002;22:184-95. https://doi. org/10.1038/sj.jp.7210687

2. Larcher V, Craig F, Bhogal K, Wilkinson D, Brierley J. Making decisions to limit treatment in life-limiting and life-threatening conditions in children: a framework for practice. Arch Dis Child. 2015;100 (Suppl 2):s1-26. https://doi.org/10.1136/ archdischild-2014-306666

3. Parravicini E. Neonatal palliative care. Curr Opin Pediatr. 2017;9:135-14. https://doi. org/10.1097/MOP.0000000000000464

4. Royal College of Paediatrics and Child Heath (RCPCH). Withholding or withdrawing life saving treatment in children. A framework for practice. London, England: RCPCH; 1997.

5. Winkler EC, Hiddemann W, Marckmann G. Evaluating a patient's request for lifeprolonging treatment: an ethical framework. J Med Ethics. 2012;38:647-51. https://doi. org/10.1136/medethics-2011-100333

6. The United Nations Children's Fund. London: UNICEF UK. [cited 2018 January 18]. Available from: https://downloads. unicef.org.uk/wp-content/uploads/2010/05/ UNCRC_united_nations_convention_on_ the_rights_of_the_child.pdf 